\title{
Comparative Study on Functional Properties of Starch Extracted from Various Sources
}

\author{
Hibu Odi and V. R. Sinija* \\ Food Processing Business Incubation Centre, Indian Institute of Food Processing \\ Technology, Thanjavur, (TN), India \\ *Corresponding author
}

\section{Keywords}

Functional properties, Tapioca starch, Rice starch, Sorghum starch, $\mathrm{pH}$, Color

Article Info

Accepted: 25 December 2020 Available Online: 10 January 2021
Starch was extracted from traditional black rice and sorghum and compared with commercial starch from tapioca for physicochemical and functional properties. The starch from rice and sorghum was extracted using the alkali method. The results showed that all the starch samples have significantly different functional properties, $\mathrm{pH}$, and color. The swelling power and solubility of tapioca starch were found to be highest, ranging from 10.50 to $30.10 \mathrm{gg}^{-1}$ and 7.35 to $27.20 \%$ (at temperature ranging from 65 to $95^{\circ} \mathrm{C}$ ), followed by sorghum and rice starch. For bulk and tapped density of the sample were in the order: sorghum starch $>$ rice starch $>$ tapioca starch. Similarly, the water and oil absorption capacity of samples followed the order: tapioca starch $>$ sorghum starch $>$ rice starch. The $\mathrm{pH}$ values of tapioca, rice, and sorghum starch were found to be 5.35, 5.85, and 6.48 respectively. Color of all starch showed high whiteness values (L* ranging from 94.39 to 94.94 ) with slight yellowish tone (hue angle ranging from $80.28^{\circ}$ to $98.05^{\circ}$ ), and very less $a^{*}, b^{*}$, and chroma values, hence indicating that all the starch was white. On comparing rice and sorghum starch with tapioca starch, it can be concluded that the functional and physicochemical properties of rice and sorghum starch are comparable to that of tapioca starch. Hence, rice and sorghum starch can be used in place of tapioca starch as an ingredient in the preparation of various products.

\section{Introduction}

Starch is one of the important carbohydrates that is available in many food materials. It is a great source of energy for man. Starch in its pure form is odorless, tasteless, soft, and looks white. It is highly soluble in hot water but insoluble in alcohol and cold water. Starch can be simplified into two forms: one is the linear and helical polymer amylose and the other is the branched polymer amylopectin. Food having higher amylopectin content shows great swelling and gelatinization properties (Tao et al., 2019). While food containing higher amylose content shows properties of amorphous materials. Also, food having higher amylopectin, or food having lower amylose, shows a higher rate of digestion (Martens et al., 2018). Due to the presence of amylose and amylopectin, starch has unique functional and physicochemical properties, and these 
properties differ in the different food sources. Due to its unique characteristics, it is widely used in various types of industries such as paper, mining, building, pharmaceutical, and food. Starch is used mainly as a stabilizer, adhesive, gelling agent, thickener, water retention agent, and binding agent. In food industries, starch is used in soup, yogurt, puddings, jellies, cream, gum, bakery products, etc., and is also used in making foods for animals and birds such as pigs, cattle, and poultry. In this study, functional properties of starch extracted from various sources like rice and sorghum was compared with tapioca starch.

\section{Materials and Methods}

\section{Raw materials}

Raw materials were procured from the local market of Thanjavur, Tamil Nadu. The raw materials used for the study were traditional black rice and sorghum. Another material used for the study was commercially available tapioca starch which was bought from local market.

\section{Starch extraction}

Starch from traditional black rice and sorghum was extracted using the alkali method (Wijesinghe \& Gunathilake, 2020). The procedures used for the extraction of starch are as shown in Figure 1.The starch yield was calculated using the following equation.

Starch yield $(\%)=($ Final dried starch weight $\div$ Initial sample weight) $* 100$

\section{Physicochemical properties}

\section{Amylose and amylopectin}

For the preparation of standard, blank, and sample solutions, procedures used in
Abeysundara et al., (2017) were used. The absorbance of the solutions was read at the wavelength of $620 \mathrm{~nm}$. The standard curve of absorbance was plotted against amylose concentration. The percentage of amylose present in the sample was calculated using the standard curve, and amylopectin percentage was calculated using the following equation.

Amylopectin $(\%)=100-$ Amylose $(\%)$

\section{pH}

The starch solution was prepared by adding $1 \%$ of the starch sample in distilled water $(\mathrm{w} / \mathrm{v})$ at room temperature (Estrada-León et al., 2016). Then, the $\mathrm{pH}$ values were measured using a $\mathrm{pH}$ meter. The readings were taken in triplicates.

\section{Color}

The color of all starch samples was analyzed using Hunter Associates Laboratory, Inc. (Reston, Virginia, USA). For each sample, $\mathrm{L}^{*}, \mathrm{a}^{*}$, and $\mathrm{b}^{*}$ were measured, and determination of hue angle and chroma was done using the following formula (EstradaLeón et al., 2016).

$$
\begin{aligned}
& \text { Hue angle }=\tan ^{-1} \frac{b *}{a *} \\
& \text { Chroma }=\sqrt{a *^{2}+b *^{2}}
\end{aligned}
$$

\section{Functional properties}

\section{Swelling power and solubility}

Swelling power and solubility was determined according to the procedures used in Kaur et al., (2018). One gram of starch sample was added to $100 \mathrm{ml}$ of distilled water. Then, the solution was heated in a water bath at 65,75 , 85 , and $95^{\circ} \mathrm{C}$ with constant stirring for $1 \mathrm{~h}$. The heated sample was cooled for $30 \mathrm{~m}$ into 
attain the final temperature of $30^{\circ} \mathrm{C}$. Then, the cooled sample was transferred to the centrifuge tube and centrifuged at 2500 rpmfor $10 \mathrm{~min}$. The residue and supernatant were separated. The weight of residue was weighed and the readings were noted down. The supernatants were dried in a hot air oven at $110^{\circ} \mathrm{C}$ for $12 \mathrm{~h}$, and then, the dry solids were weighed and the readings were noted down. The readings were taken in triplicates. Followings are the formula used for calculating swelling power and solubility (Estrada-León et al., 2016).

Solubility $(\%)=\frac{\text { Weight of dried solids (g) }}{\text { Weight of sample (g) }} \times 1$ oo

Swelling power $(\mathrm{g} / \mathrm{g})=\frac{\text { Weight of residus }(g)}{\text { Wight of sample }(g) \text {-Weight of dried solids }(g)}$

\section{Bulk and tapped density}

Bulk density and tapped density were determined according to the procedures used in Navaf et al., (2020). Starch sample weighing $1 \mathrm{~g}$ was filled in a $10 \mathrm{ml}$ measuring cylinder, and the volume was marked as $\mathrm{V}_{1}$. Then, the measuring cylinder with the starch sample was tapped 300times. This volume was marked as $V_{2}$. Finally, the bulk and tapped densities were calculated using the following formula.

Bulk density $(\mathrm{g} / \mathrm{ml})=\frac{W}{V 1}$

Tapped density $(\mathrm{g} / \mathrm{ml})=\frac{W}{V^{2}}$

where $\mathrm{w}$ is the weight of the sample.

\section{Water and oil absorption capacity}

Water and oil absorption capacity was determined according to the procedures used in de Castro et al., (2019). One gram of sample was measured and then filled in a centrifuge tube. $10 \mathrm{ml}$ of water or oil (sunflower oil) was added to the centrifuge tubes containing the sample. The suspension was homogenized for $30 \mathrm{~min}$. Then, the homogenized sample was centrifuged at $3000 \mathrm{rpm}$ for $15 \mathrm{~min}$. The supernatant was decanted to obtain a residue. The weight of residue was measured and the readings were noted down. The readings were taken in triplicates. The water or oil absorption capacity was calculated using the following formula.

Water or oil absorption capacity $(\mathrm{g} / \mathrm{g})=$ $\frac{\text { Weight of residue }(g)}{\text { Weight of sample }(g)} \times 100$

\section{Statistical analysis}

All the readings were taken in triplicates, and the data were represented as mean \pm standard deviation. The data were analyzed in Minitab 17 using one-way ANOVA. Significant differences were determined at $\mathrm{P}<0.05$ using the Tukey test.

\section{Results and Discussion}

\section{Starch yield}

From Figure 2, it can be observed that the starch yield of traditional black rice and sorghum was found to be $50.14 \%$ and $44.78 \%$ respectively. Less yield of starch is due to lower dispersibility and solubility of glutens present in the sample, and also difficulties in filtering due to an increase in viscosity of sample slurry (Wijesinghe \& Gunathilake, 2020).

\section{Physicochemical properties}

\section{Amylose and amylopectin}

Amylose and amylopectin content of tapioca, rice, and sorghum starch were tabulated as shown in Table 1, and was observed to differ significantly $(\mathrm{p}<0.05)$. Amylose content of 
rice starch $(26.61 \%)$ was the highest, followed by sorghum starch $(19.41 \%)$ and tapioca starch (17\%).Similarly, amylopectin content of tapioca starch $(83 \%)$ was found to be the highest, followed by sorghum starch $(80.59 \%)$ and rice starch $(73.39 \%)$. A similar result of amylose and amylopectin content in tapioca starch was observed in Wijesinghe and Gunathilake (2020). These differences in amylose and amylopectin content in starch samples are due to the starch extraction method, different genotypes, and environmental factors. More amylose gives better gel strength and more amylopectin gives higher viscosity (Wijesinghe and Gunathilake, 2020). Hence, rice starch and sorghum starch can be used to improve the gel strength and viscosity of the products respectively.

Table.1 Functional and physicochemical properties of starch extracted from various sources

\begin{tabular}{|c|c|c|c|c|c|c|c|}
\hline Sample & $\begin{array}{c}\text { Bulk } \\
\text { density } \\
(\mathbf{g} / \mathbf{m l})\end{array}$ & $\begin{array}{c}\text { Tapped } \\
\text { density } \\
(\mathbf{g} / \mathbf{m l})\end{array}$ & $\begin{array}{c}\text { Water } \\
\text { absorption } \\
\text { capacity } \\
\mathbf{( g / g )}\end{array}$ & $\begin{array}{c}\text { Oil } \\
\text { absorption } \\
\text { capacity } \\
\mathbf{( g / g )}\end{array}$ & $\begin{array}{c}\text { Amylose } \\
(\mathbf{\%})\end{array}$ & $\begin{array}{c}\text { Amylopectin } \\
(\mathbf{\%})\end{array}$ & pH \\
\hline Tapioca & $0.44 \pm 0.00^{\mathrm{C}}$ & $0.56 \pm 0.00^{\mathrm{C}}$ & $0.77 \pm 0.00^{\mathrm{A}}$ & $0.99 \pm 0.01^{\mathrm{A}}$ & $17.00 \pm 0.00^{\mathrm{C}}$ & $83.00 \pm 0.00^{\mathrm{A}}$ & $5.35 \pm 0.02^{\mathrm{C}}$ \\
\hline Rice & $0.48 \pm 0.00^{\mathrm{B}}$ & $0.71 \pm 0 . .00^{\mathrm{B}}$ & $0.61 \pm 0.01^{\mathrm{C}}$ & $0.90 \pm 0.00^{\mathrm{C}}$ & $26.61 \pm 0.01^{\mathrm{B}}$ & $73.39 \pm 0.01^{\mathrm{C}}$ & $5.85 \pm 0.05^{\mathrm{B}}$ \\
\hline Sorghum & $0.59 \pm 0.00^{\mathrm{A}}$ & $0.77 \pm 0.00^{\mathrm{A}}$ & $0.64 \pm 0.01^{\mathrm{B}}$ & $0.93 \pm 0.00^{\mathrm{B}}$ & $19.41 \pm 0.01^{\mathrm{A}}$ & $80.59 \pm 0.01^{\mathrm{B}}$ & $6.48 \pm 0.08^{\mathrm{A}}$ \\
\hline
\end{tabular}

Fig.1 Flow chart of starch extraction using the alkali method

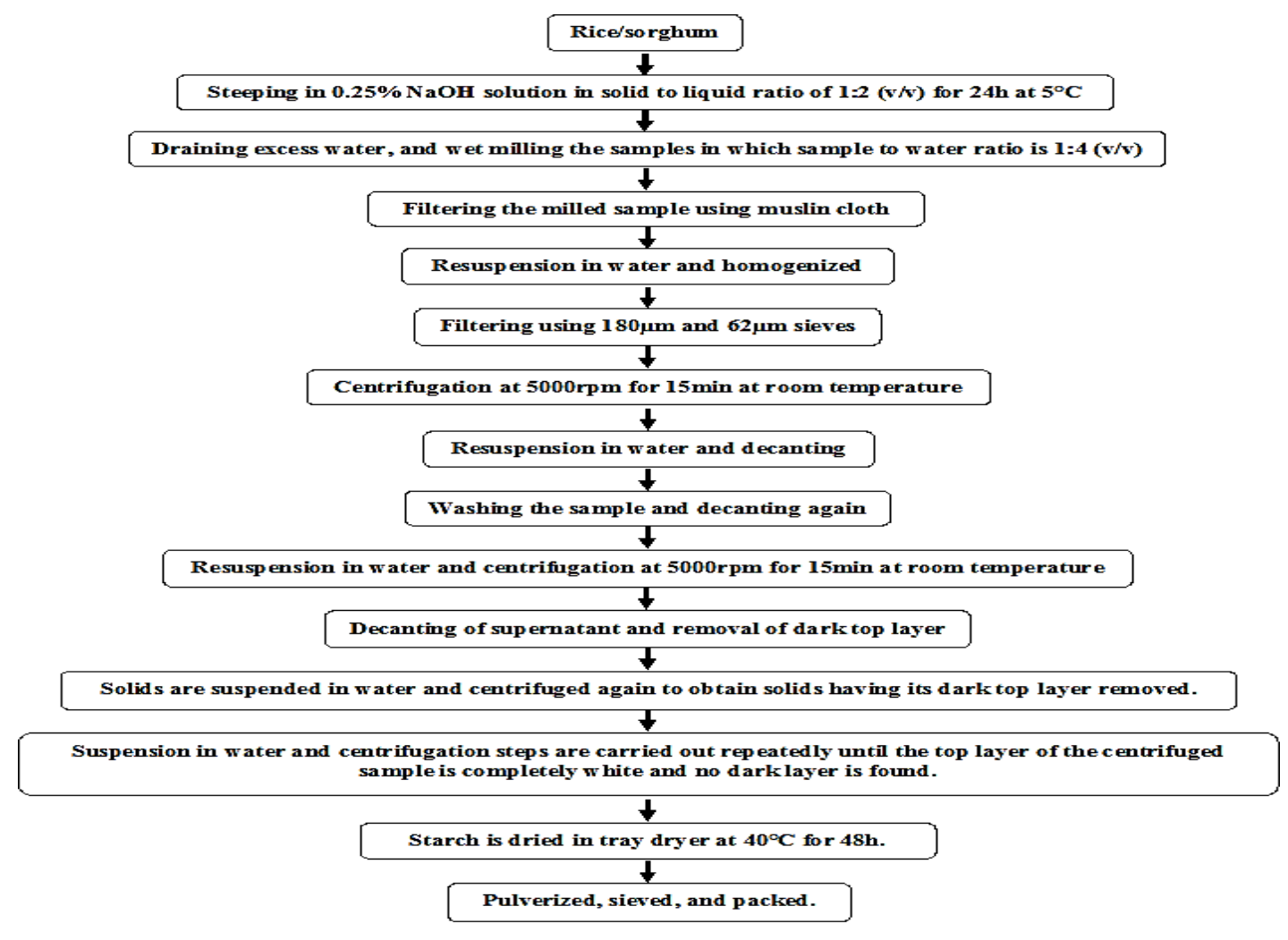


Fig.2 Yield of extracted rice and sorghum starch

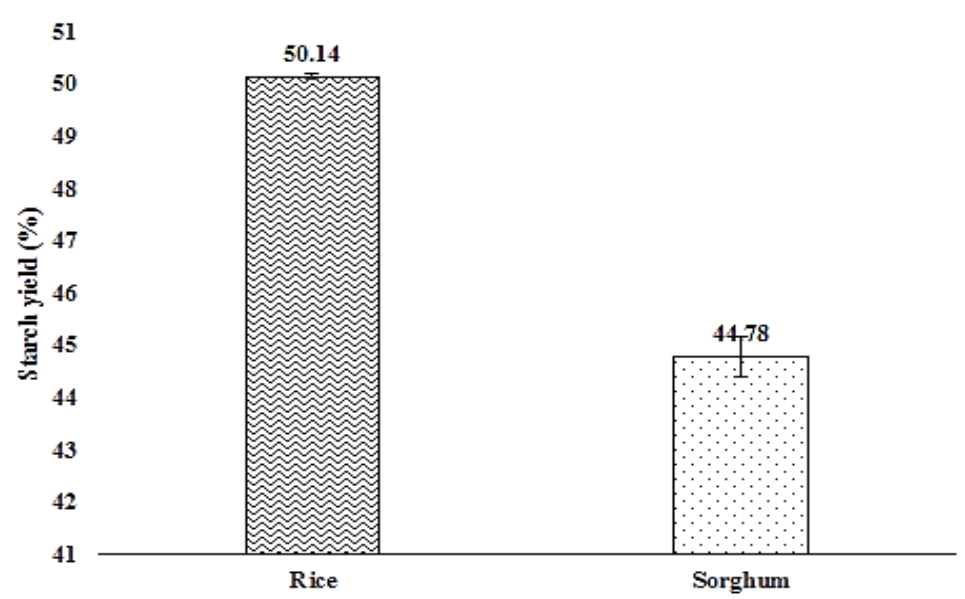

Fig.3 Color values of starch extracted from different sources

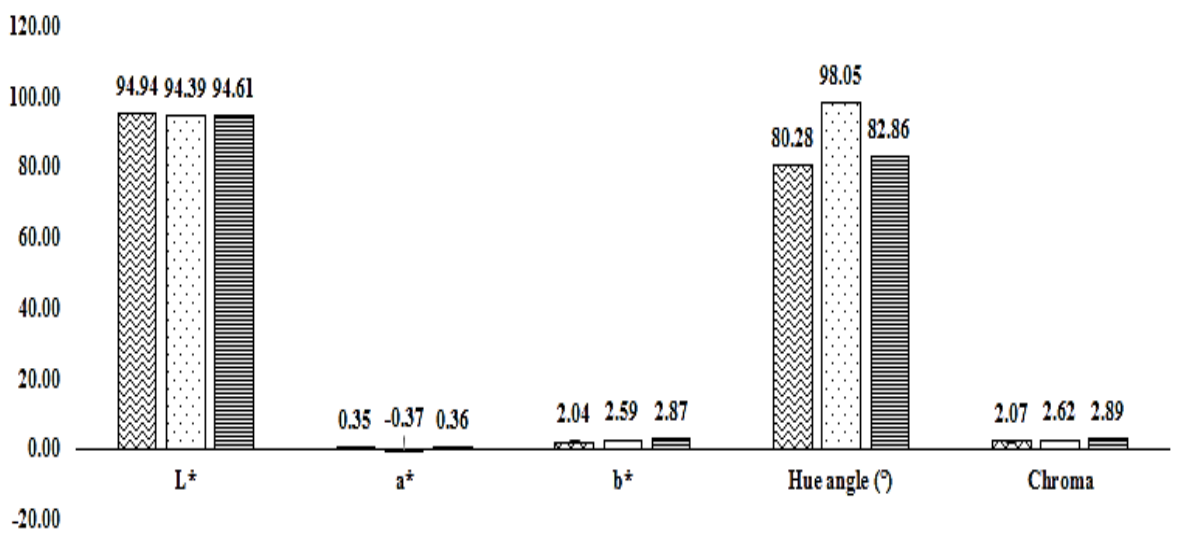

๑Rice 口Sorghum ETapioca

Fig.4 Swelling power of starch extracted from different sources at different temperatures

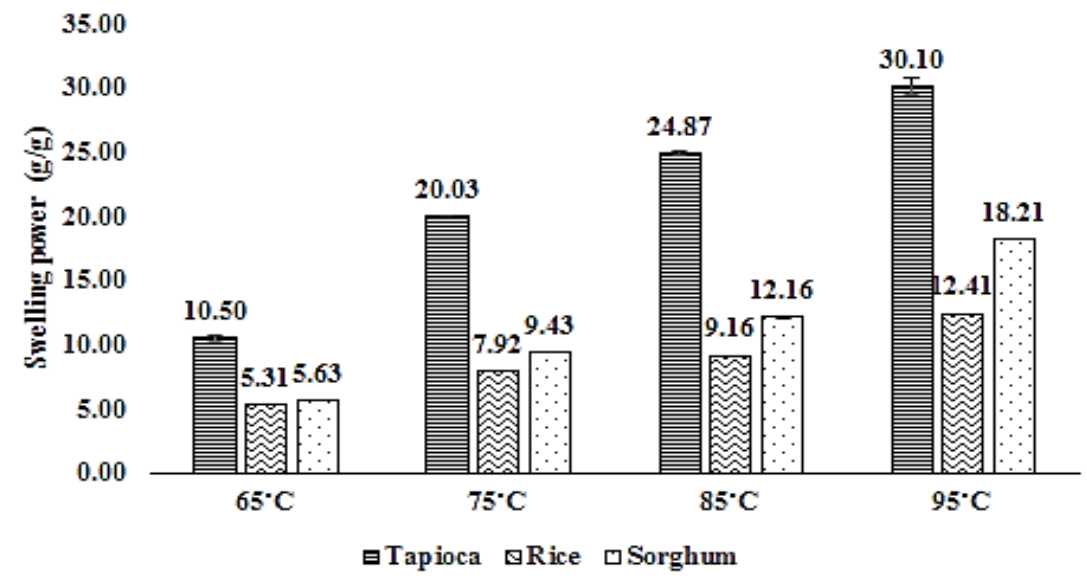


Fig.5 Solubility of starch extracted from different sources at different temperatures

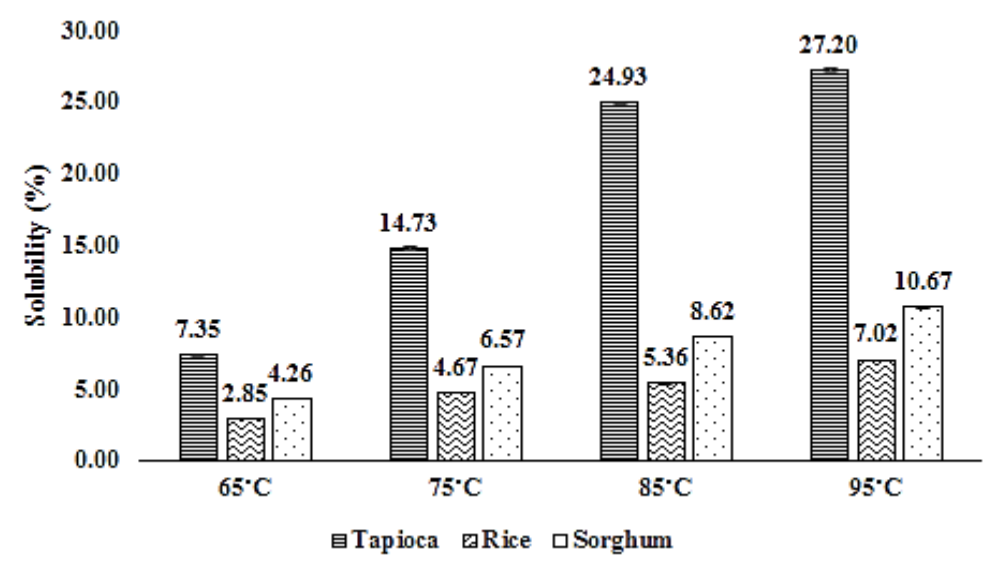

pH

From Table1, it can be observed that the $\mathrm{pH}$ values of the starch samples differed significantly $(\mathrm{p}<0.05)$. All the starch samples showed very less acidic values, i.e. 5.35, 5.85, and 6.48 for tapioca, rice, and sorghum starch respectively, inclining closer to neutrality. The $\mathrm{pH}$ of starch can act as a quality indicator (Kasaye Atlaw, 2018). Also, when the $\mathrm{pH}$ of starch is low, paste clarity is more, and vice versa (Wijesinghe and Gunathilake, 2020). Hence, the lower the $\mathrm{pH}$ value of the starch, the clearer is the paste of the starch. Therefore, paste clarity of rice and sorghum starch is less compared to tapioca starch.

\section{Color}

Figure 2 shows the color values of various starches ( $\mathrm{L}^{*}, \mathrm{a}^{*}, \mathrm{~b}^{*}$, hue angle, and chroma). $\mathrm{L}^{*}$ value of rice starch, i.e. 94.94 , is more than that of sorghum and tapioca starch, 94.39 and 94.61 respectively. This means that rice starch is whiter compared to sorghum and tapioca starch. The values of $a^{*}$ and $b^{*}$ are very less, closer to zero. From the figure, it is observed that the hue angle for sorghum starch $\left(98.05^{\circ}\right)$ is the highest, followed by tapioca starch $\left(82.86^{\circ}\right)$ and rice starch $\left(80.28^{\circ}\right)$. This means that all the starch has a yellowish tone and it is more in sorghum starch than the other starch. Also, the chroma values are very less, closer to zero. Hence, the intensity of yellowness in all the starch samples is very less and the starch tends to look whiter.

\section{Functional properties}

\section{Swelling power and solubility}

The swelling power and solubility of different starch are shown in Figure. 3 and 4 respectively. It was observed that the swelling power and solubility of the rice and sorghum starch differed significantly $(p<0.05)$ from that of tapioca starch. For all starch samples, the swelling power and solubility were increasing with the increase in temperature. Similar results were observed in Kaur et al., (2018). It can be observed from figures that swelling power and solubility of tapioca starch (ranging from 10.50 to $30.10 \mathrm{gg}^{-1}$ and 7.35 to $27.20 \%$ respectively) is more than that of rice and sorghum starch (ranging from 5.31 to $12.41 \mathrm{gg}^{-1}$ and 2.85 to $7.02 \%$, and 5.63 to $18.21 \mathrm{gg}^{-1}$ and 4.26 to $10.67 \%$, respectively). The swelling power and solubility of starch are due to the disruption of the starch structure during heating and hydrogen bonding of water molecules with the hydroxyl 
group of the starch molecules (Estrada-León et al., 2016). Hence, the more the structure disruption and hydrogen bonding, the more is the swelling power and solubility of the starch.

\section{Bulk and tapped density}

The bulk and tapped density of the starch are shown in Table 1. It can be observed that the bulk and tapped density of the starch samples differ significantly $(p<0.05)$. Bulk and tapped density of sorghum starch was observed to be highest among the starch samples, measured as $0.59 \mathrm{gml}^{-1}$ and $0.77 \mathrm{gml}^{-1}$ respectively, followed by rice and tapioca starch, i.e. $0.48 \mathrm{gml}^{-1}$ and $0.71 \mathrm{gml}^{-1}$, and $0.44 \mathrm{gml}^{-1}$ and $0.56 \mathrm{gml}^{-1}$ respectively. A similar result of bulk density of sorghum starch is observed in Hassan et al., (2015). Variation in bulk and tapped density is due to variation in particle size of the sample. Thus, the lesser the particle size, the lesser is the densities of the sample. Bulk and tapped density of the starch indicates blending and expand ability characteristics of the starch. Higher bulk and tapped density of the starch specifies better blending and expandability properties of that starch (Navaf et al., 2020). Also, in various unit operations, storage and packaging activity for starch is better with the starch having higher tapped density.

\section{Water and oil absorption capacity}

The water and oil absorption capacity of starch samples, as shown in Table 1, differed significantly $(\mathrm{p}<0.05)$. The water and oil absorption capacity of tapioca starch was observed to be highest, having the values of $0.77 \mathrm{gg}^{-1}$ and $0.99 \mathrm{gg}^{-1}$ respectively, compared to other starch, i.e. rice and sorghum starch (having $0.61 \mathrm{gg}^{-1}$ and $0.90 \mathrm{gg}^{-1}$, and $0.64 \mathrm{gg}^{-1}$ and $0.93 \mathrm{gg}^{-1}$, respectively). The higher water and oil absorption capacity of starch is due to more availability of hydrophobic and hydrophilic groups, respectively, in starch (Kasaye Atlaw, 2018). Starch with good water and oil absorption capacity can be used in various products as a thickening and stabilizing agent(de Castro et al., 2019).It can be observed from the Table that the water and oil absorption capacity of rice and sorghum starch is approximately closer to that of tapioca starch. Hence, rice and sorghum starch can also be used as a thickening and stabilizing agent in place of tapioca starch. Also, starch with good oil absorption capacity can be used to improve the mouthfeel and can be used as an oil retention agent (Kasaye Atlaw, 2018).

In conclusion the extracted starch (rice and sorghum starch) showed variations in functional and physicochemical properties compared to commercial tapioca starch. Rice starch showed high amylose content and sorghum starch showed high amylopectin content. Compared to tapioca starch, $\mathrm{pH}$ of rice and sorghum starch was more, and hence, lesser acidity. Rice starch was whiter than sorghum and tapioca starch. Swelling power and solubility of tapioca starch was more than that of rice and sorghum starch. Bulk and tapped density of rice and sorghum starch was more than the tapioca starch. Water and oil absorption capacity of rice and sorghum starch was less than the tapioca starch. However, the functional and physicochemical properties of rice and sorghum are comparable to that of tapioca starch. Hence, rice and sorghum starch can be used in place of tapioca starch in various product formulations.

\section{References}

Abeysundara, A., Navaratne, S., Wickramasinghe, I., \& Ekanayake, D. (2017). Determination of Changes of Amylose and Amylopectin Content of Paddy during Early Storage. International Journal of Science and Research (IJSR), 6(1), 2094-2097. 
https://doi.org/10.21275/art20164500

Ashwar, B. A., Gani, A., Shah, A., \& Masoodi, F. A. (2017). Physicochemical properties, invitro digestibility and structural elucidation of RS4 from rice starch. International Journal of Biological Macromolecules, 105, 471-477. https://doi.org/10.1016/j.ijbiomac.2017.07.0 57

de Castro, D. S., dos Santos Moreira, I., de Melo Silva, L. M., Lima, J. P., da Silva, W. P., Gomes, J. P., \& de Figueirêdo, R. M. F. (2019). Isolation and characterization of starch from pitomba endocarp. Food Research International, 124, 181-187. https://doi.org/10.1016/j.foodres.2018.06.03 2

Estrada-León, R. J., Moo-Huchin, V. M., RíosSoberanis, C. R., Betancur-Ancona, D., May-Hernández, L. H., Carrillo-Sánchez, F. A., Cervantes-Uc, J. M., \& Pérez-Pacheco, E. (2016). The effect of isolation method on properties of parota (Enterolobium cyclocarpum) starch. In Food Hydrocolloids (Vol. 57). Elsevier Ltd. https://doi.org/10.1016/j.foodhyd.2016.01.00 8

Hassan, E. G., Mustafa, A. M. I., \& Elfaki, A. A. (2015). Characterization and evaluation of starches from different sources. Journal of Agri-Food and Applied Sciences, 3(23116730), 101-109.

Kasaye Atlaw, T. (2018). Influence of Drying Methods on Flour Quality and Cyanide Content of Cassava Root Tuber. International Journal of Nutrition and Food Sciences, $\quad 7(4), \quad 142$. https://doi.org/10.11648/j.ijnfs.20180704.15

Kaur, H., Gill, B. S., \& Karwasra, B. L. (2018). In vitro digestibility, pasting, and structural properties of starches from different cereals. International Journal of Food Properties, 21(1), 70-85. https://doi.org/10.1080/10942912.2018.1439 955

Liu, H., Fan, H., Cao, R., Blanchard, C., \& Wang, M. (2016). Physicochemical properties and in vitro digestibility of sorghum starch altered by high hydrostatic pressure. International Journal of Biological Macromolecules, $\quad 92, \quad 753-760$. https://doi.org/10.1016/j.ijbiomac.2016.07.0 88

Martens, B. M. J., Gerrits, W. J. J., Bruininx, E. M. A. M., \& Schols, H. A. (2018). Amylopectin structure and crystallinity explains variation in digestion kinetics of starches across botanic sources in an in vitro pig model. Journal of Animal Science and Biotechnology, 9(1), 1-13. https://doi.org/10.1186/s40104-018-0303-8

Navaf, M., Sunooj, K. V., Aaliya, B., Sudheesh, C., \& George, J. (2020). Physico-chemical, functional, morphological, thermal properties and digestibility of Talipot palm (Corypha umbraculifera L.) flour and starch grown in Malabar region of South India. Journal of Food Measurement and Characterization, 14(3), 1601-1613. https://doi.org/10.1007/s11694-020-00408-1

Tao, K., Li, C., Yu, W., Gilbert, R. G., \& Li, E. (2019). How amylose molecular fine structure of rice starch affects functional properties. Carbohydrate Polymers, 204, 2431.

https://doi.org/10.1016/j.carbpol.2018.09.07 8

Wijesinghe, H. D. K. C., \& Gunathilake, K. D. P. P. (2020). Characterization and Comparison of Alkali Extracted Starches from Selected Cereals and Tubers. Asian Plant Research Journal, 5(1), 1-12. https://doi.org/10.9734/aprj/2020/v5i130096

\section{How to cite this article:}

Hibu Odi and Sinija, V. R. 2021. Comparative Study on Functional Properties of Starch Extracted from Various Sources. Int.J.Curr.Microbiol.App.Sci. 10(01): 3612-3619. doi: https://doi.org/10.20546/ijcmas.2021.1001.426 